\title{
Similarity Solutions of a Class of Perturbative Fokker-Planck Equation
}

\author{
Wen-Tsan Lin and Choon-Lin Ho \\ Department of Physics, Tamkang University \\ Tamsui 25137, Taiwan, R.O.C.
}

December 3, 2018

\begin{abstract}
In a previous work, a perturbative approach to a class of Fokker-Planck equations, which have constant diffusion coefficients and small time-dependent drift coefficients, was developed by exploiting the close connection between the Fokker-Planck equations and the Schrödinger equations. In this work, we further explore the possibility of similarity solutions of such a class of Fokker-Planck equations. These solutions possess definite scaling behaviors, and are obtained by means of the so-called similarity method.
\end{abstract}




\section{Introduction}

One of the basic equations employed to describe fluctuating macroscopic variables is the Fokker-Planck equation (FPE) [1]. It has found applications not only in physics, but also in other areas such as astrophysics [2, 3], chemistry [4, 5], biology [6, 7], finance [8], etc. In view of its wide applicability, various methods of finding exact and approximate solutions of the FPE's have been developed [1, 9, 10, 11, 12, 13, 14]. Most of the methods, however, are concerned only with FPE's with time-independent diffusion and drift coefficients. Generally, it is not easy to find solutions of FPE's with time-dependent diffusion and drift coefficients.

One of the methods of solving FPE with time-independent diffusion and drift coefficients is to transform the FPE into a time-independent Schrödinger equation, and then solve the eigenvalue problem of the latter [1, 15]. The transformation to the Schrödinger equation of a FPE eliminates the first order spatial derivative in the FPE and creates a Hermitian spatial differential operator. In [16] it was shown that this transformation can also be applied to transform FPE's, which have constant diffusion coefficients and time-dependent drift coefficients, into time-dependent Schrödinger equations. Based on this connection, a perturbative approach was developed and used to solve this special class of the FPE [16].

The diffusion equation is known to be invariant under the scale transformation $x \rightarrow \varepsilon x$, $t \rightarrow \varepsilon^{2} t$ for any scale $\varepsilon$. Thus one can find solutions, called the similarity solutions, of the diffusion equation with definite scaling behaviors by means of the so-called similarity method [17]. Such solutions were not considered in [16]. It is the purpose of this work to investigate the possibility of similarity solutions of the FPE within the perturbative framework of [16].

\section{Perturbative approach to Fokker-Planck equations}

The FPE of the probability density $W(x, t)$ in $(1+1)$-dimension is [1]

$$
\frac{\partial}{\partial t} W(x, t)=\left[-\frac{\partial}{\partial x} D^{(1)}(x, t)+\frac{\partial^{2}}{\partial x^{2}} D^{(2)}(x, t)\right] W(x, t),
$$


where $D^{(1)}(x, t)$ and $D^{(2)}(x, t)$ are the drift and diffusion coefficient respectively. The drift coefficient represents the external force acting on the particles and is usually expressed in terms of a drift potential $U(x, t)$ according to $D^{(1)}(x, t)=-\partial U(x, t) / \partial x$.

In this paper we will focus on FPE's with constant diffusion coefficients $D^{(2)}(x, t)=D>$ 0. In this case, one can solve the FPE by exploiting the connection between the FPE and the Schrödinger equation [1, 15]. Setting

$$
\psi(x, t)=\exp \left\{\frac{U(x, t)}{2 D}\right\} W(x, t)
$$

one can transfrom the FPE into the Schrödinger equation

$$
\frac{\partial \psi}{\partial t}=D \frac{\partial^{2} \psi}{\partial x^{2}}+\left(\frac{U^{\prime \prime}}{2}-\frac{U^{\prime 2}}{4 D}+\frac{\dot{U}}{2 D}\right) \psi
$$

where the prime and dot denote the derivatives with respect to $x$ and $t$, respectively. Each solution of the time-dependent Schrödinger equation gives the corresponding solution of the FPE via Eq. (2). It is, however, generally difficult to solve the Schrödinger equation exactly when the potential is time-dependent. Various approximation schemes may have to be employed.

The perturbative approach presented in [16] to solve the FPE containing a small parameter in the drift potential is summarized as follows. Suppose $U(x, t)=\sum_{n=0}^{\infty} \lambda^{n} U_{n}(x, t)$, where $|\lambda| \ll 1$ is a small parameter, and let $\psi(x, t)=\exp \{S(x, t, \lambda) / D\}$. Then from (3), we get the equation of $S(x, t, \lambda)$,

$$
\begin{aligned}
\dot{S} & =D S^{\prime \prime}+S^{\prime 2}+\bar{U} \\
\bar{U} & \equiv \frac{D}{2} U^{\prime \prime}-\frac{1}{4} U^{\prime 2}+\frac{1}{2} \dot{U} .
\end{aligned}
$$

Substituting the series form of $S(x, t, \lambda)=\sum_{n=0}^{\infty} \lambda^{n} S_{n}(x, t)$ into (4) and collecting terms of the same order of $\lambda$, one arrives at a set of differential equations that determine the functions 
$S_{n}:$

$$
\begin{aligned}
& \dot{S}_{0}=D S_{0}^{\prime \prime}+S_{0}^{\prime 2}+\left(\frac{D}{2} U_{0}^{\prime \prime}-\frac{1}{4} U_{0}^{\prime 2}+\frac{1}{2} \dot{U}_{0}\right), \\
& \dot{S}_{1}=D S_{1}^{\prime \prime}+2 S_{0}^{\prime} S_{1}^{\prime}+\left(\frac{D}{2} U_{1}^{\prime \prime}-\frac{1}{2} U_{0}^{\prime} U_{1}^{\prime}+\frac{1}{2} \dot{U}_{1}\right), \\
& \dot{S}_{2}=D S_{2}^{\prime \prime}+2 S_{0}^{\prime} S_{2}^{\prime}+S_{1}^{\prime 2}+\left(\frac{D}{2} U_{2}^{\prime \prime}-\frac{1}{2} U_{0}^{\prime} U_{2}^{\prime}-\frac{1}{4} U_{1}^{\prime 2}+\frac{1}{2} \dot{U}_{2}\right), \\
& \quad \vdots \\
& \dot{S}_{n}=D S_{n}^{\prime \prime}+\sum_{k=0}^{\infty} S_{k}^{\prime} S_{n-k}^{\prime}+\text { terms in } \bar{U} \text { of the order } \lambda^{n}, \quad n \geq 0 .
\end{aligned}
$$

With $S_{0}, S_{1}, S_{2}, \ldots$ solved, we will have an approximate solution $\psi(x, t)$, and hence of $W(x, t)$.

In this paper, we shall be interested in the drift potential $U(x, t)$ of the form $U(x, t)=$ $\lambda U_{1}(x, t)$, with all other $U_{n}=0$ for $n=0$ and $n>1$. From (2) the probability density is

$$
W(x, t)=e^{\frac{1}{D}\left(S-\frac{U}{2}\right)}=e^{\frac{S_{0}}{D}} e^{\frac{1}{D}\left(\sum_{n=1}^{\infty} \lambda^{n} S_{n}-\lambda \frac{U_{1}}{2}\right)},
$$

We assume the initial profile of $W(x, t)$ of the unperturbed case (the diffusion case) to be the delta-function, i.e., $W(x, t) \rightarrow \delta(x)$ at $t=0$ as $\lambda \rightarrow 0$. Then the solution $S_{0}(x, t)$ is [16]

$$
S_{0}(x, t)=-\frac{D}{2} \ln (4 \pi D t)-\frac{x^{2}}{4 t}
$$

leading to the probability density

$$
W_{0}(x, t)=e^{\frac{S_{0}}{D}}=\frac{1}{\sqrt{4 \pi D t}} e^{-\frac{x^{2}}{4 D t}} .
$$

Eq. (12) is the well-known solution of the diffusion equation with the delta function as the initial profile. It is evident that under the scale transformation $x \rightarrow \bar{x}=\varepsilon x, t \rightarrow \bar{t}=\varepsilon^{2} t$, $W_{0}(x, t)$ scales as $W_{0}(x, t)=\varepsilon W_{0}(\bar{x}, \bar{t})$.

\section{Scaling behavior}

Motivated by the scaling form of $W_{0}(x, t)$ in (12), we would like to seek similarity solutions of the perturbative FPE. To this end, let us first obtain the required scaling behaviors of $W(x, t)$ and $U(x, t)$ so that Eq. (11) is invariant under the scale transformation

$$
\bar{x}=\varepsilon^{a} x, \quad \bar{t}=\varepsilon^{b} t,
$$


where the scaling exponents $a$ and $b$ are arbitrary real parameters. Suppose $W(x, t)$ and $U(x, t)$ scale as $\bar{W}(\bar{x}, \bar{t})=\varepsilon^{\gamma} W(x, t)$ and $\bar{U}(\bar{x}, \bar{t})=\varepsilon^{d} U(x, t)$ with real exponent $\gamma$ and $d$, resepctively. Then Eq. (1) becomes

$$
\varepsilon^{-\gamma+b} \frac{\partial \bar{W}}{\partial \bar{t}}=\varepsilon^{-\gamma-d+2 a} \frac{\partial}{\partial \bar{x}}\left(\frac{\partial \bar{U}}{\partial \bar{x}} \cdot \bar{W}\right)+\varepsilon^{-\gamma+2 a} D \frac{\partial^{2} \bar{W}}{\partial \bar{x}^{2}} .
$$

For Eq.(14) to have the same form as Eq. (11), one must have $\varepsilon^{-\gamma+b}=\varepsilon^{-\gamma-d+2 a}=\varepsilon^{-\gamma+2 a}$. This implies that $d=0, b=2 a$, and $\gamma$ arbitrary. It means that when the scaling exponent of $t$ is twice that of $x$, and $U(x, t)$ is scale-invariant, then the FPE is scale invariant, and thus admits solutions $W(x, t)$ with arbitrary scaling exponent, which is dictated by the initial profile $W(x, 0)$. This result puts a constraint on the scaling property of the drift potential $U(x, t)$.

Applying these general results to Eq. (10), we see that since $U(x, t)=\lambda U_{1}(x, t)$ is scaleinvariant, so are all $S_{n}(x, t)(n \geq 1)$. The scaling of $W$ is therefore solely determined by the solution $\exp \left\{S_{0} / D\right\}$ of the non-perturbed FPE. With $S_{0}(x, t)$ given in (11), $W(x, t)$ scales as $W(x, t)=\varepsilon^{a} W(\bar{x}, \bar{t})$ (see Eq.(12)). This is in accord with the scaling of the initial profile: $W(x, 0)=\delta(x)=\varepsilon^{a} \delta(\bar{x})$. Our next step is to obtain the scale-invariant solutions for the $S_{n}$ 's. This is attained by the similarity method, which we describe below.

\section{Similarity method}

The similarity method is a very useful method for solving a partial differential equation which possesses proper scaling behavior. One advantage of the similarity method is to reduce the partial differential equation to an ordinary differential equation through some new independent variables (called similarity variables), which are certain combinations of the old independent variables. In our case, the 2nd order FPE can thus be transformed into an ordinary differential equation which may be easier to solve. We will illustrate the method by discussing the solution of $S_{1}$ below. The method applies to equations for other $S_{n}$ as well. 
With $U_{0}(x, t)=0$ and $S_{0}$ given in (11), the equation for $S_{1}$ is

$$
\dot{S}_{1}=D S_{1}^{\prime \prime}-\frac{x}{t} S_{1}^{\prime}+\frac{D}{2} U_{1}^{\prime \prime}+\frac{1}{2} \dot{U}_{1}
$$

Let $x$ and $t$ transform according to Eq. (13). Recall from previous discussions that $U_{1}$ and $S_{n}(n \geq 1)$ are scale-invariant, i.e., $U(x, t)=\bar{U}_{1}(\bar{x}, \bar{t})$ and $S_{n}(x, t)=\bar{S}_{n}(\bar{x}, \bar{t})(n \geq 1)$. In terms of these scaled variables, Eq.(15) becomes

$$
\varepsilon^{b} \frac{\partial \bar{S}_{1}}{\partial \bar{t}}=\varepsilon^{2 a} D \frac{\partial^{2} \bar{S}_{1}}{\partial \bar{x}^{2}}-\varepsilon^{b} \frac{\bar{x}}{\bar{t}} \frac{\partial \bar{S}_{1}}{\partial \bar{x}}+\varepsilon^{2 a} \frac{D}{2} \frac{\partial^{2} \bar{U}_{1}}{\partial \bar{x}^{2}}+\varepsilon^{b} \frac{1}{2} \frac{\partial \bar{U}_{1}}{\partial \bar{t}} .
$$

We demand that $\bar{S}_{1}$ satisfies Eq.(15) and this requires $b=2 a$. To determine the form of the similarity solution, we have to first determine a new variable $z(x, t)$ that is invariant under the scale transformation Eq.(13). From $b=2 a$, we can choose the scale-invariant similarity variable to be

$$
z=\frac{x}{\sqrt{t}}
$$

Since both $S_{1}(x, t)$ and $U_{1}(x, t)$ are scaling invariant, they must be functions of $z$ only. Hence it is reasonable to express $S_{1}(x, t)$ and $U_{1}(x, t)$ in terms of similarity variable $z$ as

$$
S_{1}(x, t)=y(z) ; \quad U_{1}(x, t)=u(z)
$$

Eq.(15) can then be cast into an ordinary differntial equation

$$
y^{\prime \prime}(z)-\frac{z}{2 D} y^{\prime}(z)+\frac{1}{2} u^{\prime \prime}(z)-\frac{z}{4 D} u^{\prime}(z)=0
$$

Eq. (19) is the most general equation for $S_{1}$ with an arbitrary scale-invariant potential $U(x, t)=\lambda U_{1}(x, t)$. Once $y(z)$ is solved, the similarity solution $S_{1}(x, t)$ of Eq.(15) is obtained by putting $z=x / \sqrt{t}$. To illustrate the procedure, we shall solve the case of a class of simple scale-invariant potentials.

\section{$5 \quad U_{1}(x, t)=\mu x^{p} t^{q}$}

For definiteness we consider the simplest form that may have the required scaling property, namely, $U_{1}(x, t)=\mu x^{p} t^{q}$ where $\mu, p$ and $q$ are arbitrary real parameters. Requiring that 
$U_{1}$ be scale-invariant under the transformation (13) with $b=2 a$, we must have $q=-p / 2$. From (18), we have

$$
u(z)=U_{1}(x, t)=\mu x^{p} t^{q}=\mu z^{p} .
$$

Eq.(19) is then expressed as

$$
y^{\prime \prime}-\frac{z}{2 D} y^{\prime}=-\frac{\mu}{2} p(p-1) z^{p-2}+\frac{\mu p}{4 D} z^{p}
$$

The general solution of Eq.(21) is

$$
y(z)=\alpha_{0} \int^{z} \exp \left\{\frac{z^{\prime 2}}{4 D}\right\} d z^{\prime}+\alpha_{1}-\frac{\mu}{2} z^{p} .
$$

In order for the probability density $W(x, t)$ to be normalizable, we require that $y(z)$ be finite as $z \rightarrow \pm \infty$. As such, $\alpha_{0}$ has to be set to zero, as the exponential term is divergent in the domain of $z \in(-\infty, \infty)$. Thus the acceptable solution is

$$
y(z)=\alpha_{1}-\frac{\mu}{2} z^{p} .
$$

Putting $z=x / \sqrt{t}$ back into (23) gives the solution of the first order perturbation equation:

$$
S_{1}(x, t)=\alpha_{1}-\frac{\mu}{2}\left(\frac{x}{\sqrt{t}}\right)^{p}
$$

With $S_{1}(x, t)$ given in (24), the equation for $S_{2}(x, t)$, i.e. Eq.(8)

$$
\dot{S}_{2}=D S_{2}^{\prime \prime}-\frac{x}{t} S_{2}^{\prime}
$$

By the procedure described in Sect. 4, this equation can be reduced to

$$
y^{\prime \prime}-\frac{z}{2 D} y^{\prime}=0
$$

As discussed before, the finite solution is $y(z)=\alpha_{2}=$ constant. Hence $S_{2}$ is given by $S_{2}(x, t)=\alpha_{2}$.

With $S_{1}$ and $S_{2}$ given above, the equations for $S_{n}(n \geq 3)$ turn out to have the same form as that for $S_{2}$, i.e., Eq. (25). Therefore the solutions for $S_{n}(n \geq 3)$ are $S_{n}(x, t)=\alpha_{n}=$ constant. 
From (10), the general solution $W(x, t)$ to FPE in the case $U_{1}=\mu x^{p} t^{q}$, where $q=-p / 2$, is

$$
\begin{aligned}
W(x, t) & \propto \exp \left\{-\frac{x^{2}}{4 D t}-\frac{\lambda \mu}{D}\left(\frac{x}{\sqrt{t}}\right)^{p}+\frac{1}{D}\left(\lambda \alpha_{1}+\lambda^{2} \alpha_{2}+\lambda^{3} \alpha_{3}+\cdots\right)\right\} \\
& \propto \exp \left\{-\frac{x^{2}}{4 D t}-\frac{\lambda \mu}{D}\left(\frac{x}{\sqrt{t}}\right)^{p}\right\} .
\end{aligned}
$$

In the last step, the constants $\alpha_{1}, \alpha_{2}, \cdots$, are set to zero since the part $\exp \left\{\frac{1}{D}\left(\lambda \alpha_{1}+\right.\right.$ $\left.\left.\lambda^{2} \alpha_{2}+\lambda^{3} \alpha_{3}+\cdots\right)\right\}$ can be absorbed into the normalization constant, which however can not be determined until the parameters $p$ and $\mu$ are given. Furthermore, for $W(x, t)$ to be normalizable, $p$ can only take the values $p=0,1,2$, and $p=$ even integer for $p>2$.

Below we shall consider two special but interesting cases of such a form of drift potentials.

\section{$5.1 \quad U_{1}=x / \sqrt{t}, p=1$ case}

Let us now consider the case with $\mu=1$ and $p=1$. The FPE is

$$
\frac{\partial W}{\partial t}=D \frac{\partial^{2} W}{\partial x^{2}}+\frac{\lambda}{\sqrt{t}} \frac{\partial W}{\partial x} .
$$

Its similarity solution is

$$
W(x, t)=\frac{1}{\sqrt{4 \pi D t}} \exp \left\{-\frac{1}{4 D t}(x+2 \lambda \sqrt{t})^{2}\right\}
$$

This result is the same as that in [16]. One can see from (29) that position $x$ is shifted as time $t$ elapses.

\section{$5.2 \quad U_{1}=x^{2} / 2 t, p=2$ case}

The second example is the case with $U_{1}(x, t)=x^{2} / 2 t$, where the parameters taken are $\mu=$ $1 / 2$ and $p=2$. From the discussions given before, we find that the probability distribution function

$$
W(x, t)=\sqrt{\frac{1+2 \lambda}{4 \pi D t}} \exp \left\{-\frac{x^{2}}{4 D t}(1+2 \lambda)\right\}
$$


is the solution to the FPE

$$
\frac{\partial W}{\partial t}=D \frac{\partial^{2} W}{\partial x^{2}}+\frac{\lambda x}{t} \frac{\partial W}{\partial x}+\frac{\lambda}{t} W
$$

In (30), one can see that the perturbation parameter $\lambda$ controls the half width of the Gaussian distribution $W(x, t)$.

\section{Summary}

When a partial differential equation possesses certain scaling behavior, the so-called similarity method is of great help in finding its solutions. One advantage of the similarity method is to reduce the partial differential equation to an ordinary differential equation through some new independent variables (called similarity variables), which are certain combinations of the old independent variables. The solutions so obtained, called similarity solutions of the differential equations, likewise possess proper scaling forms. A well-known example is the diffusion equation.

In this paper, we have applied the similarity method to a class of perturbative FPE's with small time-dependent drift potentials studied in [16]. We have presented the main ideas of the similarity method. The method was then applied to find similarity solutions of the FPE with certain scale-invariant drift potentials. Our results show that similarity method can be a useful tool to solve FPE with time-dependent drift and diffusion coefficients. While the present work is only concerned with the perturbative FPE, it can be extended to the general FPE without much difficulty [19].

\section{Acknowledgments}

This work is supported in part by the National Science Council (NSC) of the Republic of China under Grants NSC-99-2112-M-032-002-MY3 and NSC-99-2811-M-032-012. 


\section{References}

[1] H. Risken, The Fokker-Planck Equation (2nd. ed.) (Springer-Verlag, Berlin, 1996).

[2] S. Nayakshin and F. Melia, Self-consistent Fokker-Planck Treatment of Particle Distributions in Astrophysical Plasmas, Astrophysical Journal Supplement Series 114, 269 (1998).

[3] G. G. Howes, S. C. Cowley, W. Dorland, G. W. Hammett, E. Quataert and A. A. Schekochihin, Astrophysical Gyrokinetics: Basic Equations and Linear Theory, Astrophysical Journal 651, 590 (2006).

[4] B. Nowakowski, Solution of the Fokker-Planck equation for reactive Rayleigh gas, Phys. Rev. E 53, 2964 (1996).

[5] Ivan L'Heureux, Reaction rate kernel for dichotomous noise-induced transitions in bistable systems, Phys. Rev. E 51, 2787 (1995).

[6] M. Schienbein and H. Gruler, Langevin equation, Fokker-Planck equation and cell migration, Bulletin of Mathematical Biology, Vol. 55, Issue 3, 585 (1993).

[7] T. D. Frank, P. J. Beek, and R. Friedrich, Fokker-Planck perspective on stochastic delay systems: Exact solutions and data analysis of biological systems, Phys. Rev. E 68, 021912 (2003).

[8] R. Friedrich, J. Peinke and Ch. Renner, How to Quantify Deterministic and Random Influences on the Statistics of the Foreign Exchange Market, Phys. Rev. Lett. 84, 5224 (2000).

[9] R. Blackmore and B. Shizgal, Discrete-ordinate method of solution of Fokker-Planck equations with nonlinear coefficients, Phys. Rev. A 31, 1855 (1985).

[10] A. N. Malakhov and A. L. Pankratov, Exact solution of Kramers' problem for piecewise parabolic potential profiles, Physica A 229, 109 (1996). 
[11] T. Blum and A.J. McKane, Variational schemes in the Fokker-Planck equation, J. Phys. A: Math. Gen. 29, 1859 (1996).

[12] G. Hu, Solvable model of the Fokker-Planck equation without detailed balance, Phys. Rev. A 39, 1286 (1989).

[13] E. W. Larsen, C. D. Levermore, G. C. Pomraning and J. G. Sanderson, Discretization methods for one-dimensional Fokker-Planck operators, J. of Com. Phy. 61, 359 (1985).

[14] H. P. Langtangen, A general numerical solution method for Fokker-Planck equations with applications to structural reliability, Probabilistic Engineering Mechanics, Vol. 6, $33(1991)$.

[15] C.-L. Ho and R. Sasaki, Quasi-exactly solvable Fokker-Planck equations, Ann. Phys. 323,883 (2008).

[16] C.-L. Ho and Y.-M. Dai, A Perturbative Approach to a Class of Fokker-Planck Equations, Mod. Phys. Lett. B 22, 475 (2008).

[17] D. Zwillinger, Handbook of Differential Equations (2nd. ed.) (Academic Press, 1992).

[18] A. D. Polyanin and V. F. Zaitsev, Handbook of Exact Solutions for Ordinary Differential Equations (2nd. ed.) (Chapman and Hall, 2002).

[19] W.-T. Lin and C.-L.Ho, Similarity solutions of Fokker-Planck Equations (manuscript in preparation). 